\title{
A narrative review of individualized treatments of genitourinary tumors: is the future brighter with molecular evaluations?
}

\author{
Francesca Giunchi ${ }^{1}$, Tania Franceschini ${ }^{2}$, Michelangelo Fiorentino ${ }^{2}$ \\ ${ }^{1}$ Azienda Ospedaliero-Universitaria di Bologna, Bologna, Italy; ${ }^{2}$ Metropolitan Department of Pathology, University of Bologna, Bologna, Italy \\ Contributions: (I) Conception and design: M Fiorentino, F Giunchi; (II) Administrative support: None; (III) Provision of study materials or patients: \\ All authors; (IV) Collection and assembly of data: F Giunchi, T Franceschini; (V) Data analysis and interpretation: None; (VI) Manuscript writing: \\ All authors; (VII) Final approval of manuscript: All authors. \\ Correspondence to: Michelangelo Fiorentino, MD, PhD. Pathology Service, Maggiore Hospital, University of Bologna, Via dell'Ospedale 8, 40133 \\ Bologna, Italy. Email: michelangelo.fiorentino@unibo.it.
}

\begin{abstract}
Few molecular prognostic and predictive biomarkers have been identified so far in genitourinary tumors. We started from a literature search to explore the status of the art of molecular pathology tests as diagnostic, prognostic, predictive biomarkers in genitourinary cancers. Next generation sequencing approaches now provide mind-changing information in the fields of kidney cancer diagnosis, predictive oncology of urothelial cancer, understanding the causes of testicular and penile cancer, and the comprehension of the drivers of prostate cancer progression beyond androgen regulation. The classification of kidney cancer will be based soon on molecular changes. The causes of non-HPV related penile cancer are largely unknown. The emerging high incidence of testicular cancer could be explained only on the basis of molecular changes. The response to novel therapeutic agents in prostatic and urothelial cancer will require thorough molecular tumor characterization. The hereditary risk of patients with early onset prostate cancer and their potential treatment with targeted therapy requires germline and somatic genetic assays. The implementation of effective biomarkers for the response to immune check-point inhibitors in genitourinary cancer is based on the assessment of inflammatory expression profiles and the tumor mutational burden. This review deals with the current tests and provides a tentative foresee of the future molecular biomarkers of genitourinary cancer.
\end{abstract}

Keywords: Molecular pathology; precision medicine; genitourinary tumors

Submitted Aug 18, 2020. Accepted for publication Oct 19, 2020.

doi: $10.21037 /$ tau-20-1185

View this article at: http://dx.doi.org/10.21037/tau-20-1185

\section{Background}

Personalized medicine is the frontier of oncological treatments in this new century. Molecular characterization is currently mandatory in most human malignancies for precise diagnosis and to predict response to targeted biological drugs. Genitourinary cancers are particular in the landscape of solid tumors since few molecular prognostic and predictive biomarkers have been identified so far in these tumor types. In fact, the diagnosis of prostate cancer still largely relies on histology, while the therapeutic advances in lethal prostate cancer still mainly involve hormone therapy and in a small proportion of cases drugs targeting single germline genetic alterations. The molecular classification of urothelial cancer has been a major advancement in understanding the biology of this cancer type. However, the hands-on application of this molecular classification is still under-recognized by most pathologists and the predictive significance of molecular biomarkers in cancer therapy is poorly understood except for immunotherapy. Kidney cancer represents the most rapidly evolving field among solid tumors in terms of change in subtype classification. The next classification of the world health organization will encounter a large number 
of additional kidney cancer types based on molecular distinctive features as it happened to the previous edition in 2016. This improved understanding of kidney tumors with different molecular traits and shared histological features will likely impact the diagnostic and surgical approach to kidney lesions but will not probably change the therapeutic strategy of advanced/metastatic cases. Testicular cancer is facing a new epidemiological outbreak and a new biological classification is linking similar histology to a different carcinogenetic pathway and age of occurrence. Molecular advances in testicular cancer might therefore affect mainly diagnosis since oncologic therapy is standardized and associated with good clinical responses in most metastatic cases. Finally, penile cancer is a niche in both pathology and oncology and it has been mainly related to human papilloma virus (HPV) infections. The main molecular interest is focusing on non-HPV related tumors and the complex relations between cancer and the associated inflammatory microenvironment.

The rationale of this review is to start from a literature search and explore the status of the art of the impact of molecular pathology tests as diagnostic, prognostic, predictive biomarkers in genitourinary cancers. The main objective is to foresee the benefits that molecular tests will bring to the patients harboring these tumor types. We present the following article in accordance with the Narrative Review reporting checklist (available at http:// dx.doi.org/10.21037/tau-20-1185).

\section{Methods}

We performed a review of PubMed/Medline in June 2020 for the previous ten years using the following search items: genitourinary tumors and molecular classification (286 results); genitourinary tumors and molecular diagnosis (1,042 results) genitourinary tumors and molecular biomarkers (1,232 results); genitourinary tumors and therapy biomarkers (3,127 results). We applied a selection diagram to the results according to the Preferred Reporting Items for Systematic Review and Metaanalysis (PRISMA) (1). After further filtering to exclude: (I) experimental studies in animals; (II) studies on gynecological cancers; (III) generic studies on solid tumors including genitourinary cancers; (IV) review articles; (V) case reports, 383 publications were selected for inclusion in this analysis. The most significant reports have been utilized as the base for the commentary of the following two topics: molecular classification and diagnosis of genitourinary tumors; molecular prognostic and predictive biomarkers in genitourinary tumors.

\section{Molecular classification and diagnosis of genitourinary tumors}

The classification of kidney neoplasms has changed in the last 10 years to include several new entities, previously underestimated, characterized by similar features at $\mathrm{H} \& \mathrm{E}$ staining but with totally different genetic basis and clinical behavior. The recognition of these new entities that started with the meeting of the International Society of Uropathology in Vancouver in 2009 and has been recognized in the WHO classification of $2016(2,3)$.

The diagnosis of several of these new entities derived from a better molecular tumor characterization. For instance, the molecular distinctive trait of the clear cell papillary from conventional clear cell cancer is the absence of $V H L$ alterations and now this histotype is commonly diagnosed on the basis of the $\mathrm{CK}^{+}{ }^{+}$racemase ${ }^{-} \mathrm{CA}-\mathrm{IX}^{-}$ immunohistochemical algorithm $(4,5)$. The diagnosis of other renal cell tumors with papillary morphology such as the tumors associated with the succinate dehydrogenase $(S D H B)$ or the fumarate hydratase $(F H)$ deficiency has been possible after the clarification of the role of the Krebs cycle genes alterations in renal cell tumors and it is easily possible with specific immunohistochemical stains $(6,7) . X ; 11$ and 6;11 translocation tumors associated with the MIT family genes (TFE3 and TFEB) fusions represent a well-defined group of pediatric and adult kidney tumors whose diagnostic final confirmation is made possible by break-apart FISH or next generation sequencing methods $(8,9)$. The number of new tumor kidney entities is steadily growing and will be likely recognized by the new WHO classification of genitourinary tumors. Among these new entities the tumors associated with alterations of the TSC-mTOR pathway are particularly relevant (10). The so-called eosinophilic solid and cystic renal cell cancer is part of this tumor group and it is characterized by mutations in the TSC2 gene, distinctive immunoreactivity for cytokeratin 20, and favorable clinical behavior (11). Besides, several other renal tumor types are encountered in patients harboring alterations of the tuberous sclerosis complex. For instance, renal cancer with clear cell morphology and prominent muscle stroma or high-grade tumors with eosinophilic and chromophobelike features often display mutations in TSC1, TSC2 and $m$ mOR genes $(12,13)$. The increasing use of next generation sequencing panels in solid tumors leads to the discovery 
of unusual genetic alterations in tumor that would be otherwise classified as papillary renal cell carcinoma (RCC). This is the case of the renal tumors associated with ALK translocations, a molecular trait that represent a target of therapy in lung cancer. These tumors display translocations of $A L K$ with a large spectrum of partner genes including TPM3, STRN, VCL, HOOK, CLIP1 and KIFSB (14).

Non-muscle invasive urothelial cancer may present as low-grade papillary or high-grade flat lesions. From the biological standpoint both lesions seem to share the loss of the CDKN2 tumor suppressor gene encoding for the cellcycle regulator p16 protein. While FGFR3 mutations drive the growth of low-grade lesions, p53 alterations are typical of flat carcinoma in situ (CIS) $(15,16)$. The diagnosis of other superficial urothelial neoplasms such as the lesions with the typical inverted architecture have been supported by mutation of the MAP kinase/ERK pathway, while papillary urothelial neoplasms of low malignant potential (PUNLMP) are frequently related to promoter mutations of the telomerase TERT gene $(17,18)$. TERT alterations do not occur in reactive urothelial lesion and this can be helpful in distinguishing low-grade neoplastic lesions from hyperplastic proliferations such florid cystic cystitis (19). The molecular classification of muscle-invasive bladder cancer represents one of the major recent innovations in the field of urothelial cancer. Several different laboratories in the last decade reached the same conclusions about the molecular subtypes of bladder cancer that has been recognized by The Cancer Genome Atlas (TCGA) consortium (20). Starting from a strange similarity with breast cancer, these gene expression-based classification recognizes the following molecular sub-types of urothelial cancer: (I) luminal tumors (as in the breast) mostly express genes of urothelial differentiation such as GATA3 and uroplakin and frequently display mutations in FGFR3 (16). (II) Basal-squamous tumors tend to express opposite genes typical of basal cells such as p63 or proteins as cytokeratins $5 / 6$ and display variable mutations of $p 53$. (III) A third, quite undefined, subtype had been named "p53-like", "luminal infiltrated" and it is characterized by retained $p 53$ function and moderate to intense inflammatory infiltrate (20). (IV) The neuronal-like subtype identifies small-cell neuroendocrine bladder tumors. In the attempt to replicate the molecular classification using phenotypical markers pathologists have tried to use immunohistochemistry as surrogate of gene expression. Several phenotypic panels including GATA3, p63, CK5/6 and uroplakin have been proposed but none has reached the introduction in the clinical routine diagnostic practice so far $(21,22)$.

Testicular cancer most likely derives from defective maturation of primordial germ cells and it is characterized by variable cytogenetic chromosome copy number gains/ losses. The new WHO classification of testicular germcell tumors (TGCT) has re-grouped TGCTs into two categories according to the association with concomitant germ cell neoplasia in situ (GCNIS) and the age of onset. Type I and type 3 tumors such as pre-pubertal teratoma or spermatocytic seminoma do not originate from GCNIS while the vast majority of TGCTs such as seminoma, embryonal carcinoma, yolk-sac tumor and teratoma arising from GCNIS (3). The molecular hallmark of GCNIS is the gain of chromosome $12 p$, often leading to an isochromosome $\mathrm{i}(12 \mathrm{p})$. From a diagnostic view-point the assessment of the $\mathrm{i}(12 \mathrm{p})$ is helpful in distinguishing TGCT in the metastatic setting and particular in teratoma that developed a somatic malignancy, or to differentiate pre-pubertal versus post-pubertal type teratoma (23).

About half of penile cancers are associated with HPV infection (24). The WHO in 2016 has introduced a classification for penile cancer and pre-cancer based on the histological lesions associated or not with HPV infection (25). Since most of the HPV-related pre-cancer lesions progressing to invasive penile cancer are flat [HPVrelated high-grade penile intraepithelial neoplasia (PeIN)] and are associated with high-risk HPV strains, the positive immunostaining for p16 is now widely used as a surrogate for HPV molecular testing by PCR or in situ hybridization (26,27). HPV- and p16-negative penile cancers represent a peculiar epidemiological subset of tumors (28). These tumors are supposed to develop in a background of chronic inflammation and progress through hyperplastic and welldifferentiated histological lesions (differentiated PeIN). The molecular alterations at the basis of differentiated PeIN are currently unknown.

The role of molecular test for assisting the diagnosis of prostate cancer is limited to selected tumor settings. One is the identification of a prostatic primary origin in the setting of a multi-metastatic cancer dissemination at the disease's onset. Immunohistochemical markers of prostatic origin such as the tumor suppressor gene NKX3.1 or prostatespecific antigen (PSA) are reliable in most cases (29). Notwithstanding, in certain anatomical sites (serosal cancer effusions or bone metastases) and in rare cases these markers can turn under-expressed or negative and histology may be overlapping. Molecular tests such as the identification of the TMPRSS2-ERG fusion, typical of at least $50 \%$ 
Table 1 Novel biomarkers of possible future implementation in genitourinary cancer

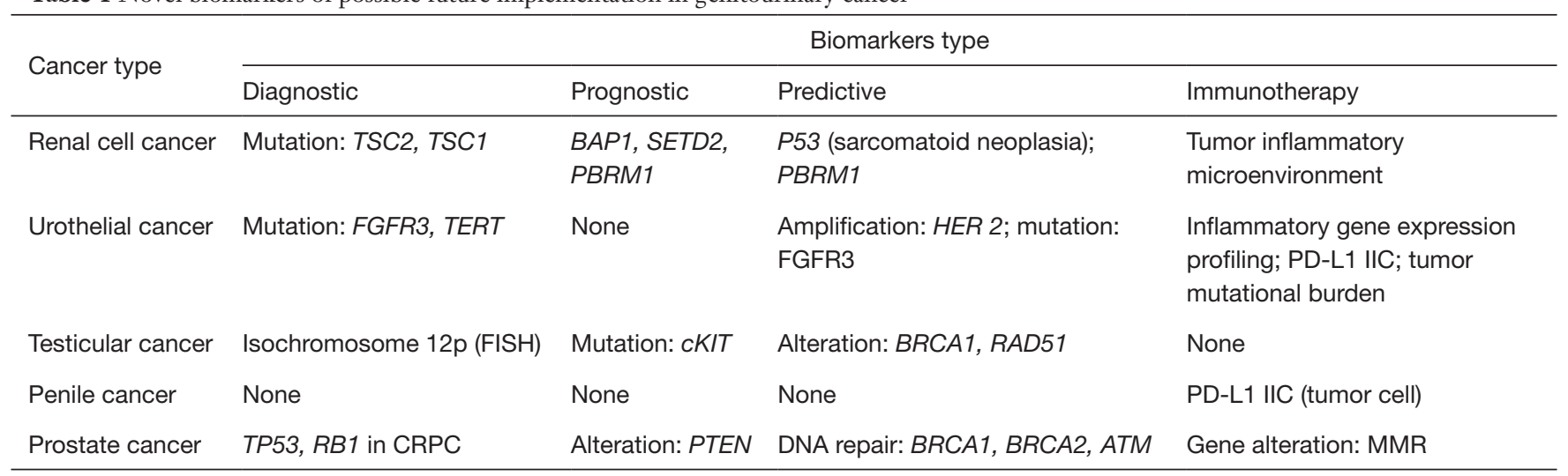

CRPC, castration resistant prostate cancer; MMR, mismatch repair.

prostate cancer or the amplification of the androgen receptor gene might be useful for diagnosis $(30,31)$. The differential diagnosis between advanced prostate cancer with neuroendocrine (NE) differentiation versus primary small-cell NE cancer of the prostate represents another diagnostic dilemma for pathologists. Both these tumor types are likely associated with clinical resistance to therapeutic hormone castration and the immunohistochemical markers of NE or prostatic differentiation are often of little help (32). Unfortunately, both primary small-cell NE carcinoma of the prostate and prostate cancers with endocrine differentiation may express the same NE immunohistochemical markers and the morphology alone does not warrant the diagnosis in all cases. The identification of molecular markers such as the inactivation of TP53 or RB1, although not entirely specific, would help better separate castration resistant prostate cancer (CRPC) from small-cell NE neoplasms with important clinical implications (33). The increasing application of next-generation sequencing (NGS) technologies to advanced prostate cancer patients for therapeutic options (see below) will certainly improve also the diagnostics of the spectrum of NE prostatic lesions (34). Novel diagnostic molecular tests are summarized in Table 1.

\section{Molecular prognostic and predictive biomarkers in genitourinary tumors}

Oncologic therapy of metastatic RCC is currently based on tyrosine kinase inhibitors (TKIs) or inhibitors of the m-TOR pathway or immune check-point inhibitors (ICPI) (35). TKIs for RCC mainly deploy an antiangiogenic consequence of the VHL inactivation in clear cell tumors through the simultaneous inhibition of several kinases including VEGFR, PDGFRA and MET. Personalized therapy of advanced RCC with TKIs is not guided by molecular tests to date. However, the use of NGS panels in RCC has recently disclosed alterations in novel genes such as PBRM1, BAP1 and SETD2, all located close to the genetic locus of VHL on chromosome $3 \mathrm{p}$. These genes are involved in chromatin remodeling and histone methylation. BAP1 and SETD2 mutations are associated with aggressive disease while PBRM1 mutations seem to confer favorable prognosis (36). ALKtranslocated RCC and pure sarcomatoid RCC represent two specific disease settings. The first unveils the potential application of anti-ALK TKIs as demonstrated in single case reports (37). The second is associated with a high frequency of TP53 alterations that leads to likely resistance to TKIs and favors the use of chemotherapy or immunotherapy in metastatic sarcomatoid cases (38). Immunotherapy with ICPI represents a major advance in the therapy of metastatic RCC with both clear-cell and non-clear-cell histology (39). The assessment of PD-L1 in RCC to predict response to ICPI is currently discouraged due to several limitations including the different antibodies and the different scoring systems (40). Additional predictors of response to ICPI including tumor mutational burden and assessment of the tumor inflammatory microenvironment are potentially promising but require prospective validations as biomarkers (41).

The molecular classification of invasive urothelial cancer has important therapeutic implications. In fact, advanced urothelial cancer of basal subtype is associated with higher response rates to cisplatin-based chemotherapy compared 
to the other subtypes (42). Similarly, a distinctive trait of the " $\mathrm{p} 53$-like" subtype is the resistance to cisplatin-based regimens (43). The luminal subtype of urothelial cancer is characterized by mutations in FGFR3. Although the number of muscle-invasive cancers of the luminal type is lower compared to the other subtypes the presence of FGFR3 alterations could be target of specific anticancer agents such as the pan-FGFR inhibitor Erdafitinib and Infigratinib. In a phase II clinical trial Erdafitinib was associated with $40 \%$ objective tumor responses in $40 \%$ of patients with urothelial cancer harboring FGFR mutations (44). Although the micropapillary variant of urothelial cancer is typically enriched in HER2 amplification the attempt to apply the well-known HER2 targeting agents used in breast cancer to urothelial malignancies has provided quite disappointing results (45). Nevertheless, the over-expression of HER2 in bladder cancer is variable, is not only restricted to micropapillary tumors and the HER2 amplification is not always coupled with protein immunoreactivity (46). Therefore, new clinical trials testing anti-HER2 agents with different patients' selection criteria are required. Predictive biomarkers for immunotherapy with ICPI in urothelial cancer would require a separate review. Currently, the two PD-1/PD-L1 inhibitors Pembrolizumab and Atezolizumab are approved for first-line treatment of advanced urothelial cancer, and other ICPI such as Durvalumab, Avelumab and Nivolumab are approved for second-line (47). Approximately 20-30\% of the patients display objective responses and some are long-lasting. Responses are more frequent among the basal and the p53-like immune-enriched molecular subtypes compared to the luminal type. As previously discussed for kidney cancer, the predictive test is based on the immunoreactivity for PD-L1 using different antibodies and different scoring systems and criteria according to each ICPI, weakening the role of immunohistochemistry as predictive biomarker (48). Molecular predictive factors of response to ICPI are more promising and are essentially based on the tumor mutation burden, inflammatory genes expression profiling and genomic instability (49). A recent trial has highlighted the synergy of combined proportional score for PD-L1 in immunohistochemistry plus tumor mutational burden in advanced urothelial cancer with predominant histological variants treated with neo-adjuvant Pembrolizumab (50). Clustering of inflammatory genes expression by gene set enrichment analyses recently turned out as a significant prognosticator in advanced urothelial cancer and was also used as a predictor of response in neo- adjuvant immunotherapy (51-53). It seems very likely that in the near future a combination of gene expression profiling for inflammatory genes, tumor mutational burden and the immunohistochemical expression of PD-L1 in inflammatory cells will be required before decision on neoadjuvant immunotherapy in urothelial cancer.

The general good prognosis of post-pubertal testicular germ cell tumors (GCTs) and the excellent response to chemotherapy even in the advanced cases has limited the application of molecular analyses in testicular cancer. The Cancer Genome Atlas investigations on GCTs highlighted a large amount of cytogenetic alterations and a very low mutational load (54). The higher rate of mutations involves seminoma where the presence of KIT genetic variants seems associated with more intense inflammatory infiltrate and potentially regressions. On the contrary, KIT wildtype seminomas seem more prone to development towards other GC histotypes (54). Chemotherapy refractory GCTs represent a small subset of patients with few residual therapeutic options. The presence of alterations in BRCA1 or RAD51 in these patients discloses the potential option for a treatment with poly (ADP-ribose) polymerase (PARP) inhibitors (55).

Investigations on kinase pathways in penile cancer evidenced activation of the PTEN, STAT3, GNRH, IL-8 and $\mathrm{B}$ cell receptor signaling and gene overexpression of GNRH, NF-кB, STAT3, ERBB2 and 3 (56). However, no TKIs have been approved to date in penile cancer. Besides target therapy, the high rates of PD-L1 expression in squamous penile cancer, the strong correlation between PD-L1 immunoreactivity in primary tumors and metastases, and the high immunogenicity of the E6 and E7 HPV proteins make immunotherapy a feasible alternative therapeutic option with several ongoing trials $(57,58)$.

PTEN and the consequent activation of the PI3K/AKT signaling pathway can be considered the most reliable biomarker of progression in prostate cancer (59). PTEN is usually lost in PCA due to deletions, truncations or inactivating mutations. Regardless of the mechanism the loss of PTEN function is invariably associated with PCA recurrence after surgery and PCA specific death $(60,61)$. DNA repair deficiency represents the cutting edge of predictive biomarkers in PCA. Genes involved in the homologous recombination including BRCA1/2 and ATM are enriched in lethal PCA and associated with a higher chance of developing resistance to castration in advanced cases $(62,63)$. In this view, the PARP inhibitor Olaparib was able to improve the disease-free survival of CRPC patients 
showing at least one alteration in BRCA1/2 or $A T M$ after failure of hormone therapy (64). Mismatch repair (MMR) is also impaired and enriched in advanced and histologically aggressive PCA compared to localized disease (65). Most PCA patients with MMR belong to Lynch families or develop sporadic mutations in the MMR genes. Although immunotherapy is not yet a therapeutic option for PCA it might represent a promising alternative in advancer patients with MMR alterations (66). Finally, the role of androgen receptor amplification and splicing variants has been deeply investigated in the last decade and associated to resistance to hormone blockade $(67,68)$. Despite the emerging evidences linking $\mathrm{AR}$ alterations to resistance to castration the tests for the assessment of AR amplification and ARv7 expression did not enter yet in the clinical practice. New prognostic and predictive biomarkers are depicted in Table 1 .

Wide genome analyses and mostly the TCGA initiative have shed light on the multi-faced genetic mechanisms behind the cancerogenesis and progression of genitourinary tumors. NGS technologies nowadays represent a simple and quite inexpensive tool to dissect the molecular alterations of single cancers. Genitourinary tumors represent the new frontier of the molecularly driven therapies and immunotherapies. The future is bright, we just need to look at it with critical eyes.

\section{Acknowledgments}

Funding: None.

\section{Footnote}

Provenance and Peer Review: This article was commissioned by the Guest Editors (Rodolfo Montironi, Alessia Cimadamore, Antonio Lopez-Beltran, Marina Scarpelli and Liang Cheng) for the series "Update on Molecular Classification and Individualized Treatments of Genitourinary Tumors" published in Translational Andrology and Urology. The article has undergone external peer review.

Reporting Checklist: The authors have completed the Narrative Review reporting checklist. Available at http:// dx.doi.org/10.21037/tau-20-1185

Conflicts of Interest: All authors have completed the ICMJE uniform disclosure form (available at http://dx.doi. org/10.21037/tau-20-1185). The series "Update on Molecular Classification and Individualized Treatments of
Genitourinary Tumors" was commissioned by the editorial office without any funding or sponsorship. The authors have no other conflicts of interest to declare.

Ethical Statement: The authors are accountable for all aspects of the work in ensuring that questions related to the accuracy or integrity of any part of the work are appropriately investigated and resolved.

Open Access Statement: This is an Open Access article distributed in accordance with the Creative Commons Attribution-NonCommercial-NoDerivs 4.0 International License (CC BY-NC-ND 4.0), which permits the noncommercial replication and distribution of the article with the strict proviso that no changes or edits are made and the original work is properly cited (including links to both the formal publication through the relevant DOI and the license). See: https://creativecommons.org/licenses/by-nc-nd/4.0/.

\section{References}

1. Moher D, Shamseer L, Clarke M, et al. Preferred reporting items for systematic review and meta-analysis protocols (PRISMA-P) 2015 statement. Syst Rev 2015;4:1.

2. Srigley JR, Delahunt B, Eble JN, et al. The International Society of Urological Pathology (ISUP) Vancouver classification of renal neoplasia. Am J Surg Pathol 2013;37:1469-89.

3. Moch H, Humphrey PA, Ulbright TM, et al. eds. WHO Classification of Tumours of the Urinary System and Male Genital Organs. Lyon, France: International Agency for Research on Cancer, 2016:18-21.

4. Williamson SR, Eble JN, Cheng L, et al. Clear cell papillary renal cell carcinoma: differential diagnosis and extended immunohistochemical profile. Mod Pathol 2013;26:697-708.

5. Giunchi F, Gruppioni E, Altimari A, et al. Similarities and Differences between Clear Cell Tubulo-Papillary and Conventional Clear Cell Renal Cell Carcinoma: A Comparative Phenotypical and Mutational Analysis. Diagnostics (Basel) 2020;10:123.

6. Gill AJ, Hes O, Papathomas T, et al. Succinate dehydrogenase (SDH)-deficient renal carcinoma: a morphologically distinct entity: a clinicopathologic series of 36 tumors from 27 patients. Am J Surg Pathol 2014;38:1588-602.

7. Trpkov K, Hes O, Agaimy A, et al. Fumarate hydratasedeficient renal cell carcinoma is strongly correlated 
with fumarate hydratase mutation and hereditary leiomyomatosis and renal cell carcinoma syndrome. Am J Surg Pathol 2016;40:865-75.

8. Argani P, Ladanyi M. Renal carcinomas associated with Xp11.2 translocations/TFE3 gene fusions. In: Eble JN, Sauter G, Epstein JI, et al. eds. Pathology and Genetics of Tumours of the Urinary System and Male Genital Organs. Lyon, France: IARC Press, 2004:37-8.

9. Green WM, Yonescu R, Morsberger L, et al. Utilization of a TFE3 break-apart FISH assay in a renal tumor consultation service. Am J Surg Pathol 2013;37:1150-63.

10. Chen YB, Mirsadraei L, Jayakumaran G, et al. Somatic mutations of TSC2 or MTOR characterize a morphologically distinct subset of sporadic renal cell carcinoma with eosinophilic and vacuolated cytoplasm. Am J Surg Pathol 2019;43:121-31.

11. Trpkov K, Hes O, Bonert M, et al. Eosinophilic, solid, and cystic renal cell carcinoma: clinicopathologic study of 16 unique, sporadic neoplasms occurring in women. Am J Surg Pathol 2016;40:60-71.

12. Jia L, Jayakumaran G, Al-Ahmadie H, et al. Expanding the morphologic spectrum of sporadic renal cell carcinoma (RCC) harboring somatic TSC or MTOR alterations: analysis of 8 cases with clear cytoplasm and leiomyomatous stroma. Mod Pathol 2019;32:78-9.

13. He, H, Trpkov K, Martinek P, et al. "High-grade oncocytic renal tumor": morphologic, immunohistochemical, and molecular genetic of 14 cases. Virchows Arch 2018;473:725-38

14. Kuroda N, Liu Y, Tretiakova M, et al. Clinicopathological study of seven cases of ALK-positive renal tumor identification of new fusion partners including CLIP1 and KIF5B genes. Mod Pathol 2019;32:85.

15. McKenney JK. Precursor lesions of the urinary bladder. Histopathology 2019;74:68-76.

16. Downes MR, Weening B, van Rhijn BW, et al. Analysis of papillary urothelial carcinomas of the bladder with grade heterogeneity supportive evidence for an early role of CDKN2A deletions in the FGFR3 pathway. Histopathology 2017;70:281-9.

17. Isharwal S, Hu W, Sarungbam J, et al. Genomic landscape of inverted urothelial papilloma and urothelial papilloma of the bladder. J Pathol 2019;248:260-5.

18. Kurtis B, Zhuge J, Ojaimi C, et al. Recurrent TERT promoter mutations in urothelial carcinoma and potential clinical applications. Ann Diagn Pathol 2016;21:7-11.

19. Cheng L, Davidson DD, Wang M, et al. Telomerase reverse transcriptase (TERT) promoter mutation analysis of benign, malignant and reactive urothelial lesions reveals a subpopulation of inverted papilloma with immortalizing genetic change. Histopathology 2016;69:107-13.

20. Kamoun A, de Reyniès A, Allory Y, et al. A Consensus Molecular Classification of Muscle-invasive Bladder Cancer. Eur Urol 2020;77:420-33.

21. Sjödahl G. Molecular Subtype Profiling of Urothelial Carcinoma Using a Subtype-Specific Immunohistochemistry Panel. Methods Mol Biol 2018;1655:53-64.

22. Rebola J, Aguiar P, Blanca A, et al. Predicting outcomes in non-muscle invasive $(\mathrm{Ta} / \mathrm{T} 1)$ bladder cancer: the role of molecular grade based on luminal/basal phenotype. Virchows Arch 2019;475:445-55.

23. Idrees MT, Ulbright TM, Epstein JI. Fluorescent In Situ Hybridization Analysis for 12p Alterations in Sarcomatoid Yolk Sac Tumors. Am J Surg Pathol 2019;43:1566-73.

24. Alemany L, Cubilla A, Halec G, et al. Role of human papillomavirus in penile carcinomas worldwide. Eur Urol 2016;69:953-61.

25. Cubilla AL, Dillner J, AminMB, et al. Tumours of the penis. In: Moch H, Humphrey PA, Ulbright TM, et al. eds. World Health Organization Classification of Tumours of the Urinary System and Male Genital Organs, 4th ed. Lyon, France: IARC, 2016:259-86.

26. Olesen TB, Sand FL, Rasmussen CL, et al. Prevalence of human papillomavirus DNA and p16INK4a in penile cancer and penile intraepithelial neoplasia: a systematic review and meta-analysis. Lancet Oncol 2019;20:145-58.

27. Cubilla AL, Lloveras B, Alejo M, et al. Value of p16(INK)4(a) in the pathology of invasive penile squamous cell carcinomas: a report of 202 cases. Am J Surg Pathol 2011;35:253-61.

28. Soskin A, Vieillefond A, Carlotti A, et al. Warty/ basaloid penile intraepithelial neoplasia is more prevalent than differentiated penile intraepithelial neoplasia in nonendemic regions for penile cancer when compared with endemic areas: a comparative study between pathologic series from Paris and Paraguay. Hum Pathol 2012;43:190-6.

29. Gurel B, Ali TZ, Montgomery EA, et al. NKX3.1 as a marker of prostatic origin in metastatic tumors. Am J Surg Pathol 2010;34:1097-105.

30. Williamson SR, Zhang S, Yao JL, et al. ERG-TMPRSS2 rearrangement is shared by concurrent prostatic adenocarcinoma and prostatic small cell carcinoma and absent in small cell carcinoma of the urinary bladder: evidence supporting monoclonal origin. Mod Pathol 
2011;24:1120-7.

31. Qu X, Randhawa G, Friedman C, et al. A three-marker FISH panel detects more genetic aberrations of AR, PTEN and TMPRSS2/ERG in castration-resistant or metastatic prostate cancers than in primary prostate tumors. PLoS One 2013;8:e74671.

32. Bellur S, Van der Kwast T, Mete O. Evolving concepts in prostatic neuroendocrine manifestations: from focal divergent differentiation to amphicrine carcinoma. Hum Pathol 2019;85:313-27.

33. Tan HL, Sood A, Rahimi HA, et al. Rb loss is characteristic of prostatic small cell neuroendocrine carcinoma. Clin Cancer Res 2014;20:890-903.

34. Rubin MA, Demichelis F. The Genomics of Prostate Cancer: A Historic Perspective. Cold Spring Harb Perspect Med 2019;9:a034942.

35. Ciccarese C, Brunelli M, Montironi R, et al. The prospect of precision therapy for renal cell carcinoma. Cancer Treat Rev 2016;49:37-44.

36. Randall JM, Millard F, Kurzrock R. Molecular aberrations, targeted therapy, and renal cell carcinoma: current stateof-the-art. Cancer Metastasis Rev 2014;33:1109-24.

37. Pal SK, Bergerot P, Dizman N, et al. Responses to Alectinib in ALK-rearranged Papillary Renal Cell Carcinoma. Eur Urol 2018;74:124-8.

38. Wang Z, Kim TB, Peng B, et al. Sarcomatoid Renal Cell Carcinoma Has a Distinct Molecular Pathogenesis, Driver Mutation Profile, and Transcriptional Landscape. Clin Cancer Res 2017;23:6686-96.

39. Grimm MO, Leucht K, Grünwald V, et al. New First Line Treatment Options of Clear Cell Renal Cell Cancer Patients with PD-1 or PD-L1 Immune-Checkpoint Inhibitor-Based Combination Therapies. J Clin Med 2020;9:565.

40. Kammerer-Jacquet SF, Deleuze A, Saout J, et al. Targeting the PD-1/PD-L1 Pathway in Renal Cell Carcinoma. Int J Mol Sci 2019;20:1692.

41. Zhu J, Armstrong AJ, Friedlander TW, et al. Biomarkers of immunotherapy in urothelial and renal cell carcinoma: PD-L1, tumor mutational burden, and beyond. J Immunother Cancer 2018;6:4.

42. Seiler R, Ashab HAD, Erho N, et al. Impact of Molecular Subtypes in Muscle-invasive Bladder Cancer on Predicting Response and Survival after Neoadjuvant Chemotherapy. Eur Urol 2017;72:544-54.

43. Choi W, Porten S, Kim S, et al. Identification of distinct basal and luminal subtypes of muscle-invasive bladder cancer with different sensitivities to frontline chemotherapy. Cancer Cell 2014;25:152-65.

44. Loriot Y, Necchi A, Park SH, et al. Erdafitinib in Locally Advanced or Metastatic Urothelial Carcinoma. N Engl J Med 2019;381:338-48.

45. Powles T, Huddart RA, Elliott T, et al. Phase III, DoubleBlind, Randomized Trial That Compared Maintenance Lapatinib Versus Placebo After First-Line Chemotherapy in Patients with Human Epidermal Growth Factor Receptor 1/2-Positive Metastatic Bladder Cancer. J Clin Oncol 2017;35:48-55.

46. Franceschini T, Capizzi E, Massari F, et al. Immunohistochemical over-expression of HER2 does not always match with gene amplification in invasive bladder cancer. Pathol Res Pract 2020;216:153012.

47. Massari F, Di Nunno V, Cubelli M, et al. Immune checkpoint inhibitors for metastatic bladder cancer. Cancer Treat Rev 2018;64:11-20.

48. Cimadamore A, Scarpelli M, Massari F, et al. Immunotherapy for urothelial cancer: from the diagnostic pathologist's point of view. Expert Opin Biol Ther 2020;20:539-44.

49. Necchi A, Madison R, Raggi D, et al. Comprehensive Assessment of Immuno-oncology Biomarkers in Adenocarcinoma, Urothelial Carcinoma, and Squamouscell Carcinoma of the Bladder. Eur Urol 2020;77:548-56.

50. Necchi A, Raggi D, Gallina A, et al. Updated Results of PURE-01 with Preliminary Activity of Neoadjuvant Pembrolizumab in Patients with Muscle-invasive Bladder Carcinoma with Variant Histologies. Eur Urol 2020;77:439-46.

51. Tang Y, Hu Y, Wang J, et al. A novel risk score based on a combined signature of 10 immune system genes to predict bladder cancer prognosis. Int Immunopharmacol 2020;87:106851.

52. Wang Y, Ba HJ, Liu ZC, et al. Prognostic value of immune cell infiltration in bladder cancer: A gene expression-based study. Oncol Lett 2020;20:1677-84.

53. Necchi A, Anichini A, Raggi D, et al. Pembrolizumab as Neoadjuvant Therapy Before Radical Cystectomy in Patients with Muscle-Invasive Urothelial Bladder Carcinoma (PURE-01): An Open-Label, Single-Arm, Phase II Study. J Clin Oncol 2018;36:3353-60.

54. Shen H, Shih J, Hollern DP, et al. Integrated Molecular Characterization of Testicular Germ Cell Tumors. Cell Rep 2018;23:3392-406

55. Jacobsen C, Honecker F. Cisplatin resistance in germ cell tumours: models and mechanisms. Andrology 2015;3:111-21. 
56. Yang ES, Willey CD, Mehta A, et al. Kinase analysis of penile squamous cell carcinoma on multiple platforms to identify potential therapeutic targets. Oncotarget 2017;8:21710-8.

57. McGregor B, Sonpavde G. Immunotherapy for advanced penile cancer - rationale and potential. Nat Rev Urol 2018;15:721-3.

58. Gassian N, Frontczak A, Mouillet G, et al. Activity and tolerability of maintenance avelumab immunotherapy after first line polychemotherapy including platinum in patients with locally advanced or metastatic squamous cell penile carcinoma: PULSE. Bull Cancer 2020;107:eS16-eS21.

59. Shorning BY, Dass MS, Smalley MJ, et al. The PI3KAKT-mTOR Pathway and Prostate Cancer: At the Crossroads of AR, MAPK, and WNT Signaling. Int J Mol Sci 2020;21:4507.

60. Lotan TL, Wei W, Morais CL, et al. PTEN Loss as Determined by Clinical-grade Immunohistochemistry Assay Is Associated with Worse Recurrence-free Survival in Prostate Cancer. Eur Urol Focus 2016;2:180-8.

61. Ahearn TU, Pettersson A, Ebot EM, et al. A Prospective Investigation of PTEN Loss and ERG Expression in Lethal Prostate Cancer. J Natl Cancer Inst 2015;108:djv346.

62. Mateo J, Boysen G, Barbieri CE, et al. DNA Repair in

Cite this article as: Giunchi F, Franceschini T, Fiorentino M. A narrative review of individualized treatments of genitourinary tumors: is the future brighter with molecular evaluations? Transl Androl Urol 2021;10(3):1553-1561. doi: 10.21037/tau-201185
Prostate Cancer: Biology and Clinical Implications. Eur Urol 2017;71:417-25.

63. Na R, Zheng SL, Han M, et al. Germline Mutations in ATM and BRCA1/2 Distinguish Risk for Lethal and Indolent Prostate Cancer and are Associated with Early Age at Death. Eur Urol 2017;71:740-7.

64. de Bono J, Mateo J, Fizazi K, et al. Olaparib for Metastatic Castration-Resistant Prostate Cancer. N Engl J Med 2020;382:2091-102.

65. Abida W, Cheng ML, Armenia J, et al. Analysis of the Prevalence of Microsatellite Instability in Prostate Cancer and Response to Immune Checkpoint Blockade. JAMA Oncol 2019;5:471-8.

66. Nava Rodrigues D, Rescigno P, Liu D, et al. Immunogenomic analyses associate immunological alterations with mismatch repair defects in prostate cancer. J Clin Invest 2018;128:4441-53. Erratum in: J Clin Invest. 2018 Nov 1;128(11):5185. doi: 10.1172/JCI125184. Epub 2018 Nov 1.

67. Antonarakis ES, Lu C, Wang H, et al. AR-V7 and resistance to enzalutamide and abiraterone in prostate cancer. N Engl J Med 2014;371:1028-38.

68. Sharp A, Coleman I, Yuan W, et al. Androgen receptor splice variant-7 expression emerges with castration resistance in prostate cancer. J Clin Invest 2019;129:192-208. 